\title{
Operator product expansion and the short distance behavior of 3-flavor baryon potentials
}

\author{
Sinya Aoki \\ Graduate School of Pure and Applied Sciences, University of Tsukuba, Tsukuba, Ibaraki \\ 305-8571, Japan, and \\ Center for Computational Sciences, University of Tsukuba, Tsukuba, Ibaraki 305-8577, \\ Japan \\ E-mail: saoki@het.ph.tsukuba.ac.jp \\ Janos Balog \\ Research Institute for Particle and Nuclear Physics, 1525 Budapest 114, Pf. 49, \\ Hungary \\ E-mail: balog@rmki.kfki.hu \\ Peter Weisz \\ Max-Planck-Institut für Physik, Föhringer Ring 6, D-80805 München, Germany \\ E-mail: pew@mpp.mpg.de
}

\begin{abstract}
The short distance behavior of baryon-baryon potentials defined through Nambu-Bethe-Salpeter wave functions is investigated using the operator product expansion. In a previous analysis of the nucleon-nucleon case, corresponding to the SU(3) channels $27_{s}$ and $\overline{10}_{a}$, we argued that the potentials have a repulsive core. A new feature occurs for the case of baryons made up of three flavors: manifestly asymptotically attractive potentials appear in the singlet and octet channels. Attraction in the singlet channel was first indicated by quark model considerations, and recently been found in numerical lattice simulations. The latter have however not yet revealed asymptotic attraction in the octet channels; we give a speculative explanation for this apparent discrepancy.
\end{abstract}

KEYWORDS: Repulsive core, operator product expansion, 3-flavor baryon potential, attraction in the singlet channel. 


\section{Contents}

1. Introduction

2. 3-flavor results

3. Comparison to quark model calculations and lattice Monte Carlo 7

4. Conclusion 10

\section{Introduction}

In a recent paper [1] a proposal has been made to study nucleon-nucleon (NN) potentials from a first principle QCD approach. In this field theoretic framework, potentials are obtained through the Schrödinger operator applied to Nambu-Bethe-Salpeter (NBS) wave functions:

$$
V(\vec{x})=E+\frac{1}{2 \mu} \frac{\nabla^{2} \varphi_{E}(\vec{x})}{\varphi_{E}(\vec{x})} .
$$

Here $\mu$ is the two nucleon reduced mass and the NBS wave function is defined by

$$
\varphi_{E}(\vec{x})=\langle 0|N(\vec{x} / 2,0) N(-\vec{x} / 2,0)| 2 \mathrm{~N}, E\rangle,
$$

where $|2 \mathrm{~N}, E\rangle$ is a QCD eigenstate with energy $E$ (suppressing here other quantum numbers), and $N$ is a nucleon interpolating operator made of 3 quarks. Such wave functions have been measured through numerical simulations of the lattice regularized theory 11, 2, 3, 国. Although many conceptual questions remain to be resolved (the potential defined by (1.1) is for example energy dependent), the corresponding potentials indeed

qualitatively resemble phenomenological NN potentials which are widely used in nuclear physics. The force at medium to long distance $(r \geq 2 \mathrm{fm})$ is shown to be attractive. This feature has long well been understood in terms of pion and other heavier meson exchanges. At short distance, a characteristic repulsive core is reproduced by the lattice QCD simulation [四]. In [5] we performed an operator product expansion (OPE) analysis of $\mathrm{NN}$ NBS wave functions in QCD to theoretically better understand the repulsive core of the NN potential (at least that of the measured NBS potential). Thanks to the property of asymptotic freedom of QCD the form of leading short distance behavior of the coefficent functions can be computed using perturbation theory. To set the stage we first give a very short summary of the results of [5]. 
The behavior of the wave functions $\varphi_{E}(\vec{x})$ at short distances $(r=|\vec{x}| \rightarrow 0)$ is encoded in the operator product expansion (OPE) of the two nucleon operators:

$$
N(\vec{x} / 2,0) N(-\vec{x} / 2,0) \approx \sum_{k} D_{k}(\vec{x}) \mathcal{O}_{k}(\overrightarrow{0}, 0)
$$

where $\left\{\mathcal{O}_{k}\right\}$ is a set of local color singlet 6-quark operators with two-nucleon quantum numbers. Asymptotically the $\vec{x}$-dependence and energy dependence of the wave function is factorized into

$$
\varphi_{E}(\vec{x}) \approx \sum_{k} D_{k}(\vec{x})\left\langle 0\left|\mathcal{O}_{k}(\overrightarrow{0}, 0)\right| 2 \mathrm{~N}, E\right\rangle .
$$

Standard renormalization group (RG) analysis gives [5] the leading short distance behavior of the OPE coefficient function as

$$
D_{k}(\vec{x}) \approx\left(\ln \frac{r_{0}}{r}\right)^{\nu_{k}} d_{k},
$$

where $\nu_{k}$ is related to the 1-loop coefficient of the anomalous dimension of the operator $\mathcal{O}_{k}$, $d_{k}$ is the tree-level contribution ${ }^{1}$ of $D_{k}(\overrightarrow{0})$ and finally $r_{0}$ is some typical non-perturbative QCD scale (which we take here to be $1 \mathrm{fm}$ ). Clearly the operator with largest RG power $\nu_{k}$ dominates the wave function (1.2) at short distances. We will denote the leading (largest) power by $\nu_{1}$ and the subleading one (second largest) by $\nu_{2}$.

If neither $\nu_{1}$ nor $d_{1}$ vanishes, this leads to the leading asymptotics of the s-wave potential of the form

$$
V(r) \approx-\frac{\nu_{1}}{r^{2}\left(\ln \frac{r_{0}}{r}\right)}
$$

which is attractive for $\nu_{1}>0$ and repulsive for $\nu_{1}<0$. Note that the above asymptotic form is energy independent.

If $\nu_{1}=0$, the situation is more complicated. In this case the relative sign of the ratio $R$ between the leading contribution and the subleading contribution, corresponding to an operator $\mathcal{O}_{2}$ with $\nu_{2}<0$, is important. If $R$ is positive, the potential is repulsive, while it is attractive for negative $R$. The leading asymptotics of the potential is energy dependent in this case, since it is proportional to $R$ which depends on the energy and it may even change sign as function of $E$.

In the nucleon potential problem the above degenerate case $\left(\nu_{1}=0\right)$ occurs. In [5] we have argued that the relative coefficient $R$ is positive and the short distance limit of the nucleon potential is repulsive in both possible isospin-spin s-wave channels $(I J=01$ and $I J=10)$.

To actually compute the renormalization group power $\nu_{k}$ of an operator, we first have to compute the 1-loop coefficient of its anomalous dimension defined by [5]:

$$
\gamma_{k}(g)=\gamma_{k}^{(1)} g^{2}+\mathrm{O}\left(g^{4}\right)
$$

\footnotetext{
${ }^{1}$ In principle, the leading term might come from an operator that has vanishing tree level coefficient $d_{k}$ and enters the two-nucleon OPE at higher order in perturbation theory, provided that its anomalous dimension is large enough to compensate. In 5] we calculated the anomalous dimensions of all relevant 6-quark operators and showed that no such operators exist.
} 
Here we assume that the corresponding operator $\mathcal{O}_{k}$ is renormalized multiplicatively. It is always possible to choose an operator basis where this is the case, at least up to 1-loop level. Having computed the coefficient $\gamma_{k}^{(1)}$ the RG power $\nu_{k}$ is given by

$$
\nu_{k}=\frac{\gamma_{k}^{(1)}-2 \gamma_{N}^{(1)}}{2 \beta_{0}}=\frac{48 \pi^{2} \gamma_{k}^{(1)}-24}{66-4 N_{f}}
$$

where $\beta_{0}$ is the 1-loop beta function coefficient of the $N_{f}$ flavor theory and $\gamma_{N}^{(1)}=12 / 48 \pi^{2}$ is the 1-loop coefficient of the anomalous dimension of the nucleon operator. For simplicity we count the anomalous dimension coefficients in units of $1 / 48 \pi^{2}$ and for example we shall call an operator $\mathcal{O}_{k}$ of anomalous dimension 36 if $\gamma_{k}^{(1)}=\frac{3}{4 \pi^{2}}=\frac{36}{48 \pi^{2}}$.

In this paper we extend the analysis of ref. [5] to the case of three flavors. Instead of nucleons, we shall consider the baryon octet and study the short distance behavior of the NBS wave function

$$
\varphi_{\alpha \beta}^{i j}(\vec{x})=\left\langle 0\left|B_{\alpha}^{i}(\vec{x} / 2,0) B_{\beta}^{j}(-\vec{x} / 2,0)\right| 2 \mathrm{~B}, E\right\rangle,
$$

where $i, j$ are $\mathrm{SU}(3)$ flavor octet indices, $\alpha, \beta$ spin indices, $B_{\alpha}^{i}$ baryon field operators and $|2 \mathrm{~B}, E\rangle$ 2-baryon states with energy $E$ (and suppressed other quantum numbers). Since the operator anomalous dimensions are mass-independent in perturbation theory, all our results are manifestly SU(3) symmetric. The tensor product of two octets can be decomposed as

$$
\mathbf{8} \otimes \mathbf{8}=(\mathbf{1} \oplus \mathbf{8} \oplus \mathbf{2 7})_{s}+(\mathbf{8} \oplus \mathbf{1 0} \oplus \overline{\mathbf{1 0}})_{a},
$$

where the first three representations are symmetric, the last three antisymmetric in the two baryon indices. In terms of octet fields, for example, we have

$$
\begin{aligned}
B B^{(27)}\left(I=0, I_{3}=0, Y=0\right) & =\sqrt{\frac{27}{40}} \Lambda \Lambda-\sqrt{\frac{1}{40}} \Sigma \Sigma+\sqrt{\frac{12}{40}} N \Xi, \\
B B^{(8 s)}(0,0,0) & =-\sqrt{\frac{1}{5}} \Lambda \Lambda-\sqrt{\frac{3}{5}} \Sigma \Sigma+\sqrt{\frac{1}{5}} N \Xi, \\
B B^{(1)}(0,0,0) & =-\sqrt{\frac{1}{8}} \Lambda \Lambda+\sqrt{\frac{3}{8}} \Sigma \Sigma+\sqrt{\frac{4}{8}} N \Xi,
\end{aligned}
$$

with

$$
\begin{aligned}
& \Sigma \Sigma=\sqrt{\frac{1}{3}} \Sigma^{+} \Sigma^{-}-\sqrt{\frac{1}{3}} \Sigma^{0} \Sigma^{0}+\sqrt{\frac{1}{3}} \Sigma^{-} \Sigma^{+} \\
& N \Xi=\sqrt{\frac{1}{4}} p \Xi^{-}+\sqrt{\frac{1}{4}} \Xi^{-} p-\sqrt{\frac{1}{4}} n \Xi^{0}-\sqrt{\frac{1}{4}} \Xi^{0} n
\end{aligned}
$$

and

$$
\begin{aligned}
B B^{(\overline{10})}(0,0,2) & =\sqrt{\frac{1}{2}} p n-\sqrt{\frac{1}{2}} n p, \\
B B^{(10)}(3 / 2,3 / 2,1) & =\sqrt{\frac{1}{2}} p \Sigma^{+}-\sqrt{\frac{1}{2}} \Sigma^{+} p, \\
B B^{(8 a)}(0,0,0) & =\sqrt{\frac{1}{4}} p \Xi^{-}-\sqrt{\frac{1}{4}} \Xi^{-} p-\sqrt{\frac{1}{4}} n \Xi^{0}+\sqrt{\frac{1}{4}} \Xi^{0} n .
\end{aligned}
$$


Here $I$ and $I_{3}$ are the total isospin and its third component while $Y$ is the total hyper charge for two baryons. The spin quantum numbers are understood as in (2.5). There are six s-wave potentials corresponding to these six channels, since, due to the total antisymmetry of the two-baryon product, the flavor representation and its symmetry properties uniquely determine the spin representation for s-waves: with the antisymmetric representations the symmetric $J=1$ spin representation is coupled and $J=0$ is paired with the symmetric flavor representations. In this scheme the nucleon potentials are in the $\mathbf{2 7}$ and $\overline{\mathbf{1 0}}$ channels, for isospin-spin $I J=10$ and $I J=01$, respectively.

\section{3-flavor results}

We have repeated the analysis of [5] for the case of three quark flavors. In the three-flavor case some channels may become attractive at short distance since the Pauli exclusion principle is less significant than in the 2-flavor case. In this paper we therefore investigate which representations of the flavor $\mathrm{SU}(3)$ become attractive at short distance using the OPE. The method and the formulae necessary to compute the 1-loop anomalous dimension of any local gauge invariant 6-quark operator are given in detail in ref. [5] and will not be reproduced here.

The local gauge invariant 6-quark operators we need are local products of six quark field operators $q_{\alpha}^{f a}$ with flavor index $f \in\{u, d, s\}$, color index $a$ and Dirac index $\alpha \in\{1,2,3,4\}$ and can be written as local products

$$
T_{\alpha \beta \gamma}^{f g h} T_{\alpha^{\prime} \beta^{\prime} \gamma^{\prime}}^{f^{\prime} g^{\prime} h^{\prime}}
$$

of two color singlet triple-quark objects of the form

$$
T_{\alpha \beta \gamma}^{f g h}=\epsilon^{a b c} q_{\alpha}^{f a} q_{\beta}^{g b} q_{\gamma}^{h c} \equiv f_{\alpha} g_{\beta} h_{\gamma}
$$

where $f, g, h, f^{\prime}, g^{\prime}, h^{\prime}$ are flavor indices and $\alpha, \beta, \gamma, \alpha^{\prime}, \beta^{\prime}, \gamma^{\prime}$ Dirac indices. The Dirac matrices are chosen such that $\alpha=1,2$ correspond to positive chirality and $\alpha=3,4$ to negative chirality. As shown in [5], the 1-loop renormalization matrix mixes operators with one pair of flavor and/or Dirac indices interchanged, provided that the Dirac indices belong to the same chirality. From this it follows that operators with different flavor or Dirac index structure do not mix. It also follows that (for any flavor structure) we always have operators with vanishing $R G$ power since for the Dirac structure (the square brackets indicate antisymmetrization)

$$
T_{1[12]}^{f g h} T_{3[34]}^{f^{\prime} g^{\prime} h^{\prime}}
$$

index exchange between quark fields belonging to different triplets is not allowed by the above mentioned chirality rule and therefore the operator has anomalous dimension 24 (coming from exchange within the triplets), which is exactly compensated by the contribution of the two baryons as in (1.8).

It is sufficient to consider 6-quark operators with flavor structure uuddss since all six channels of $(1.10)$ are present in this case. There are many possible Dirac structures but there are only a few among these that contain "attractive" operators (i.e. operators with 
anomalous dimension $>24$ in our units). We have considered all Dirac structures, calculated the 1-loop anomalous dimension matrix and performed the change of basis necessary to make operators renormalize multiplicatively (diagonally) at 1-loop level. We refrain from giving a complete list here (which would be quite long: with Dirac structure 112334 for example there are 123 independent uuddss flavor 6-quark operators), and give here the complete list of attractive operators only. These are

- Dirac structure: 112334, anomalous dimension: 42 (SU(3) singlet)

- Dirac structure: 111222, anomalous dimension: 36 (SU(3) singlet)

- Dirac structure: 112234, anomalous dimension: 36 (SU(3) singlet and octet)

- Dirac structure: 111234, anomalous dimension: 32 (SU(3) singlet and octet)

- Dirac structure: 112234, anomalous dimension: 32 (SU(3) singlet and octet)

We here listed operators with chiral $(1,2)$ indices not less in number than antichiral $(3,4)$ ones. There are also parity reflected operators with the same anomalous dimension and flavor quantum numbers.

We still have to study the question whether these operators are already present at tree level in the OPE since otherwise their effective anomalous dimension is reduced by a large number (54 in our units for every extra order in perturbation theory with three flavors) and they become repulsive.

The baryon field operators corresponding to the flavor structure $f g h$ are built from the building blocks

$$
b_{\alpha L}^{f g h}=f_{\alpha}\left(g_{1} h_{2}-g_{2} h_{1}\right), \quad b_{\alpha R}^{f g h}=f_{\alpha}\left(g_{3} h_{4}-g_{4} h_{3}\right),
$$

for $\alpha=1,2,3,4$ and the physical baryon field is

$$
B_{\alpha}^{f g h}=b_{\alpha L}^{f g h}+b_{\alpha R}^{f g h}+b_{\hat{\alpha} L}^{f g h}+b_{\hat{\alpha} R}^{f g h},
$$

where $\alpha=1,2$ and $\hat{1}=3, \hat{2}=4$. We sometimes use octet indices ( $p=u u d, \Xi^{-}=s s d$, etc.) instead of the flavor notation $f g h$.

It turns out that (with the exception of the anomalous dimension 32 flavor singlet operators) all the attractive operators are indeed present at tree level in the OPE for two building blocks of the form (2.4). We find

$$
\begin{aligned}
& b_{\alpha L}^{i} b_{\hat{\beta} R}^{j}+b_{\alpha R}^{i} b_{\hat{\beta} L}^{j} \quad \approx \quad \delta^{i j} \gamma^{[42 ; 1]} K_{\alpha \beta}^{[42]}+\ldots, \\
& b_{\alpha L}^{i} b_{\beta R}^{j}+b_{\alpha R}^{i} b_{\beta L}^{j} \quad \approx \quad f^{i j k} \beta^{[32 ; 8]} L_{\alpha \beta}^{[32] k}+\delta^{i j} \beta^{[36 ; 1]} \epsilon_{\alpha \beta} L^{[36]} \\
& +d^{i j k} \beta^{[36 ; 8]} \epsilon_{\alpha \beta} L^{[36] k}+\ldots, \\
& b_{\alpha L}^{i} b_{\beta L}^{j} \quad \approx \quad \delta^{i j} \alpha_{1}^{[36 ; 1]} \epsilon_{\alpha \beta} M^{[36]}+\ldots, \\
& b_{\hat{\alpha} L}^{i} b_{\hat{\beta} L}^{j} \quad \approx \quad \delta^{i j} \alpha_{2}^{[36 ; 1]} \epsilon_{\alpha \beta} L^{[36]}+d^{i j k} \alpha^{[36 ; 8]} \epsilon_{\alpha \beta} L^{[36] k}+\ldots
\end{aligned}
$$


where ... stand for non-attractive operators. We also have the parity reflected counterparts of the above relations. We will denote the parity reflected operators with a . Here the coefficients $\gamma, \beta, \alpha$ are nonvanishing and the $K, L, M$ are local 6-quark operators with their anomalous dimension and $\mathrm{SU}(3)$ quantum numbers indicated.

Finally we can compute the baryon-baryon tree level OPE:

$$
B_{\alpha}^{i} B_{\beta}^{j} \approx \delta^{i j} \epsilon_{\alpha \beta}\left\{a_{1} \mathcal{A}_{1}^{[36]}+a_{2} \mathcal{A}_{2}^{[36]}+a_{3} \mathcal{A}_{3}^{[42]}\right\}+d^{i j k} \epsilon_{\alpha \beta} b \mathcal{B}^{[36] k}+f^{i j k} c \mathcal{C}_{\alpha \beta}^{[32] k}+\ldots
$$

Here

$$
\begin{aligned}
a_{1} & =\alpha_{1}^{[36 ; 1]}, & \mathcal{A}_{1}^{[36]} & =M^{[36]}+\hat{M}^{[36]}, \\
a_{2} & =\alpha_{2}^{[36 ; 1]}+\beta^{[36 ; 1]}, & \mathcal{A}_{2}^{[36]} & =L^{[36]}+\hat{L}^{[36]}, \\
a_{3} & =\gamma^{[42 ; 1]}, & \mathcal{A}_{3}^{[42]} & =K_{12}^{[42]}-K_{21}^{[42]}, \\
b & =\alpha^{[36 ; 8]}+\beta^{[36 ; 8]}, & \mathcal{B}^{[36] k} & =L^{[36] k}+\hat{L}^{[36] k}, \\
c & =\beta^{[32 ; 8]}, & \mathcal{C}_{\alpha \beta}^{[32] k} & =L_{\alpha \beta}^{[32] k}+\hat{L}_{\alpha \beta}^{[32] k} .
\end{aligned}
$$

We see that there are no attractive operators in the $\mathbf{2 7}_{s}, \mathbf{1 0}_{a}$ and $\overline{\mathbf{1 0}}_{a}$ channels. This is consistent with what we found in the nucleon case; these belong to $\mathbf{2 7}_{s}$ and $\overline{\mathbf{1 0}}_{a}$. There are three attractive channels: $\mathbf{1}_{s}, \mathbf{8}_{s}, \mathbf{8}_{a}$. Among these the NBS wave function for $\mathbf{1}_{s}$ becomes most singular at short distance since its anomalous dimension 42 is largest. In terms of the corresponding potential, however, all three have the same $r$ dependence at short distance. They may not be equally strong: the coefficients $a_{1}$ etc. can be calculated ${ }^{2}$ but these coefficients are to be multiplied by the matrix elements of the corresponding operators, which we do not know.

We note that there is no local 6 -quark operator in the $\mathbf{8}_{s}$ channel with nonvanishing non-relativistic limit, and therefore the corresponding matrix elements also vanish in the same limit. We considered a class of 6-quark operators in the non-relativistic limit, which have uuddss flavor and $S_{z}=0$ with $S_{z}$ being the $z$ component of the total spin. Since $q_{\alpha}=$ $q_{\hat{\alpha}}$ in the non-relativistic limit, this class of operators has 111222 Dirac structure. We found by an explicit calculation that all attractive 6 -quark operators in the $\boldsymbol{8}_{s}$ channel vanish in the non-relativistic limit. Next we calculated the eigenvalues $T$ of the SU(3) Casimir operator (and their eigenvectors) for this class of operators. The octet representation corresponds to $T=3$. We have found that while the operator $p_{1} \Xi_{2}^{-}-\Xi_{1}^{-} p_{2}$ contains $T=3$ components, the operator $p_{1} \Xi_{2}^{-}+\Xi_{1}^{-} p_{2}$ does not contain $T=3$ components at all. (The subscripts 1,2 represent spin indices.) This shows that there is no $\boldsymbol{8}_{s} 6$-quark operator in the non-relativistic limit.

One can also see the above property group theoretically [6]. The 6-quark operator must be totally antisymmetric due to the Fermi-Dirac statistics. In terms of the permutation group, the totally antisymmetric representation corresponds to $\left[1^{6}\right]$, whose Young diagram has 6 boxes in a column. Each quark operator in the non-relativistic limit has color $(c)$, $\operatorname{spin}(s)$, flavor $(f)$ in addition to the coordinate $x$ which is common for all 6 quarks in the

\footnotetext{
${ }^{2}$ e.g. at tree level $\alpha^{[36,8]} / \beta^{[36,8]}=-2 / 3$ and $\alpha_{2}^{[36,1]} / \beta^{[36,1]}=1 / 3$.
} 
local operator. We now consider symmetries of each index. The symmetry for color SU(3) must be $\left[2^{3}\right]_{c}$ due to gauge invariance, while that for the coordinate is totally symmetric, therefore $[6]_{x}$. For 3 flavor, the non-relativistic quark belongs to the fundamental representation of $\mathrm{SU}(6)_{s f} \supset \mathrm{SU}(2)_{s} \otimes \mathrm{SU}(3)_{f}$. The symmetry of 6 quarks in $\mathrm{SU}(6)_{s f}$, denoted as $[F]_{s f}$, must satisfy

$$
\left[2^{3}\right]_{c} \otimes[F]_{s f} \otimes[6]_{x} \supset\left[1^{6}\right] \Rightarrow\left[2^{3}\right]_{c} \otimes[F]_{s f} \supset\left[1^{6}\right],
$$

in order to make the 6-quark operator totally antisymmetric. In $\mathrm{SU}(6)_{s f}$, [3] contains octet and decuplet, and therefore two octet baryon state belongs to $[3] \otimes[3]=[6] \oplus[4,2] \oplus[5,1] \oplus$ $\left[3^{2}\right]$, where the first(last) two are (anti)symmetric under exchange of two [3]. Since two baryons at the same space-time point must be antisymmetric under exchange of the two, $[5,1]$ and $\left[3^{2}\right]$ are allowed. The table C on p.116 of Ref.[7] tells us

$$
\left[2^{3}\right]_{c} \otimes[5,1]_{s f}=\left[2^{2}, 1^{2}\right] \oplus[3,2,1], \quad\left[2^{3}\right]_{c} \otimes\left[3^{2}\right]_{s f}=\left[1^{6}\right] \oplus\left[2^{2}, 1^{2}\right] \oplus\left[3^{2}\right] \oplus\left[4,1^{2}\right] .
$$

These show that the $[5,1]_{s f}$ representation does not satisfy the condition $(2.12)$ while $\left[3^{2}\right]_{s f}$ does. Therefore only $\left[3^{2}\right]_{s f}$, but not $[5,1]_{s f}$, must appear in the local (gauge-invariant) 6 quark operator. In terms of $\mathrm{SU}(6)_{s f}$, the 6 quarks in the $\boldsymbol{8}_{s}$ channel, which has $\left[3^{2}\right]_{s}$ in spin and $[3,2,1]_{f}$ in flavor, becomes (see also the same table $\mathrm{C}$ )

$$
\mathbf{8}_{s}=\left[3^{2}\right]_{s} \otimes[3,2,1]_{f}=[5,1] \oplus[4,2] \oplus\left[4,1^{2}\right] \oplus 2[3,2,1] \oplus 3\left[3,1^{3}\right] \oplus\left[2^{2}, 1^{2}\right] \oplus\left[2,1^{4}\right]
$$

while $\mathbf{1}_{s}$ and $\mathbf{8}_{a}$ are expressed as

$$
\begin{gathered}
\mathbf{1}_{s}=\left[3^{2}\right]_{s} \otimes\left[2^{3}\right]_{f}=\left[3^{2}\right] \oplus\left[4,1^{2}\right] \oplus\left[2^{2}, 1^{2}\right] \oplus\left[1^{6}\right] \\
\mathbf{8}_{a}=[4,2]_{s} \otimes[3,2,1]_{f}=[5,1] \oplus 2[4,2] \oplus 2\left[4,1^{2}\right] \oplus\left[3^{2}\right] \oplus 3[3,2,1] \\
\oplus\left[2^{3}\right] \oplus 2\left[3,1^{3}\right] \oplus 2\left[2^{2}, 1^{2}\right] \oplus\left[2,1^{4}\right] .
\end{gathered}
$$

Since $\boldsymbol{8}_{s}$ contains $[5,1]$ only but no $\left[3^{2}\right]$, the 6 quark operator in this channel does not exist in the $\mathrm{SU}(6)_{s f}$ representation (i.e. the non-relativistic limit).

\section{Comparison to quark model calculations and lattice Monte Carlo}

The short distance part of the baryon potential has also been calculated using a valence quark model with gluon exchange [6] and lattice Monte Carlo measurements [8].

In the quark model above the only attractive channel is the $\mathrm{SU}(3)$ singlet one (which may correspond to a strange dibaryon bound state: the $H$ particle). It is not possible to quantitatively compare the results with ours since the way the baryon potential is defined in the two formalisms is different and in the valence quark model SU(3) breaking effects are also taken into account (and they are, in some cases, comparable to the $\mathrm{SU}(3)$ symmetric 


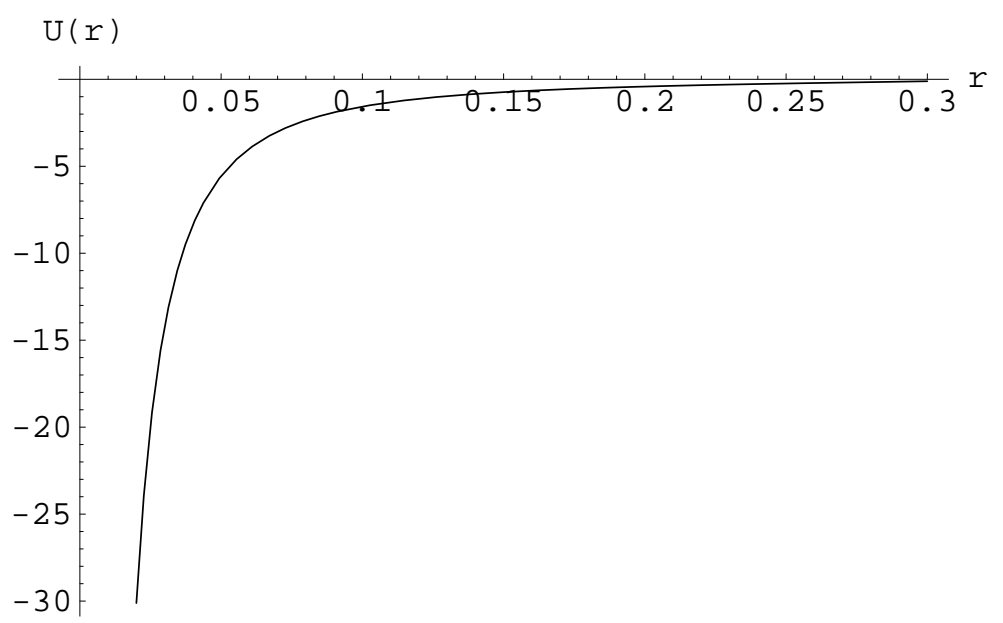

Figure 1: Potential corresponding to the model wave function with $\nu_{1}=2 / 9, \nu_{2}=-0.10$ and $R=2$.

contributions). In addition, a particle basis $(N, \Xi, \Sigma, \Lambda)$ is used in the valence quark model and the various s-wave channels are labeled as in this example:

$$
N \Sigma \quad(I, J)=\left(\frac{1}{2}, 0\right) .
$$

In the quark model this channel is for example strongly repulsive but this result cannot be directly compared to ours since in our language this is a linear combination of the channels $\mathbf{2 7}_{s}$ and $\mathbf{8}_{s}$. On the other hand, for the case

$$
N \Sigma \quad(I, J)=\left(\frac{3}{2}, 0\right)
$$

the quark model gives (a somewhat weaker) repulsion, consistently with our result, since this channel is pure $\mathbf{2 7}$.

The MC measurements 8 are performed using full QCD with three massive quarks in the flavor $\mathrm{SU}(3)$ limit. The results (for s-wave potentials) are analysed in terms of the six channels (1.10) and attraction is found only in the singlet channel. The other five are found to be repulsive, with four of them (including the $\mathbf{2 7}$ and $\overline{\mathbf{1 0}}$ already occurring in the nucleon case) being rather similar. The potential corresponding to the $\mathbf{8}_{s}$ channel is different from all the other ones in that it does not have any attractive pocket and its repulsive core is stronger and broader than for the other cases.

Our results do not necessarily contradict to what was found in the quark model and measured on the lattice since the distance range in which our asymptotic results are valid may not overlap with the range where the other two methods work. The latter is the range between $0.1 \mathrm{fm}$ and $1.5 \mathrm{fm}$, whereas the true asymptotic behavior predicted by asymptotically free perturbation theory may be valid in a much shorter distance range only and in the range where comparison is possible subleading contributions in the OPE may be non-negligible. To illustrate this situation we consider a (renormalization group motivated) simpified model where the baryon wave function is the sum of a leading term with RG power $\nu_{1}$ and a subleading term with relative coefficient $R$ and RG power $\nu_{2}$. 
More precisely, we take

$$
\psi(r)=\lambda^{-\nu_{1}}+R \lambda^{-\nu_{2}}
$$

as our simplified baryon wave function, where $\lambda(r)$ is the 2-loop 3-flavor running coupling defined by

$$
\frac{1}{\lambda}+\kappa \ln \lambda=\ln \frac{r_{0}}{r}, \quad \kappa=\frac{\beta_{1}}{2 \beta_{0}^{2}}=\frac{32}{81} .
$$

Using $\lambda(r)$ instead of $1 / \ln \left(r_{0} / r\right)$ takes into account some of the higher perturbative contributions. Of course, the complete RG asymptotic form of the NBS wave function is much more involved than the simple formula (3.3). There are perturbative corrections (power series in $\lambda$ ) multiplying both the leading power of $\lambda$ and the subleading one and there are also many sub-subleading terms to be added. Nevertheless the simple model (3.3) can qualitatively reproduce the main features of the true asymptotics of the wave function. We take

$$
\nu_{1}=\frac{2}{9}, \quad \nu_{2}=-0.1
$$

We have chosen these numbers because we want to model the $\mathbf{8}_{s}$ channel, which is asymptotically attractive but looks strongly repulsive in the range studied by lattice Monte Carlo and valence quark models. $\nu_{1}$ corresponds to anomalous dimension 36 , which is the largest (and only attractive one) occurring in this channel and $\nu_{2}$ is chosen to represent the "average" of the RG power zero (which, as we have seen, occurs in all channels) and the many negative sub-subleading powers.

Clearly for $r \rightarrow 0$ eventually the leading term dominates, but since $\nu_{1}$ and $\nu_{2}$ are both small numbers the subleading effects are still important in the range 0.1-0.3 fm, where the short distance expansion (3.3) can be compared to results obtained by the above two methods. If the relative coefficient $R$ becomes large, the second term will dominate in this range and the potential becomes repulsive. In Fig. 1 the potential ${ }^{3}$ for $R=2$ is shown. It is less attractive than the one corresponding to the leading term alone, but is still weakly attractive. On the other hand for $R=-2$ already the second term dominates and the potential, which is shown in Fig. 2, is clearly repulsive in this range.

A natural question arising is that if this mechanism explains why an asymptotically attractive potential can look repulsive in the intermediate range 0.1-0.3 fm, why the same mechanism can not modify the results obtained in the nucleon case (where repulsion was found in agreement with the quark model and Monte Carlo results). The answer is that in the nucleon case all subleading terms correspond to repulsion so if they happen to dominate in the intermediate range the qualitative behavior of the potential remains unchanged. Actually the nucleon case corresponds to the degenerate case with $\nu_{1}=0$ and the true asymptotics is determined by the sign of the relative coefficient $R$. But the above explained mechanism actually improves the situation in the intermediate range since if $R$ becomes large (with either sign), it dominates and the potential looks repulsive. Note that the potential should become attractive asymptotically at short distance for negative $R$. The attractive potential can be seen in the intermediate range only if $R \approx-1$ or smaller.

\footnotetext{
${ }^{3}$ The model potential is simply defined as $U(r)=\nabla^{2} \psi / \psi$. We are using $r_{0}$ units for the plots.
} 


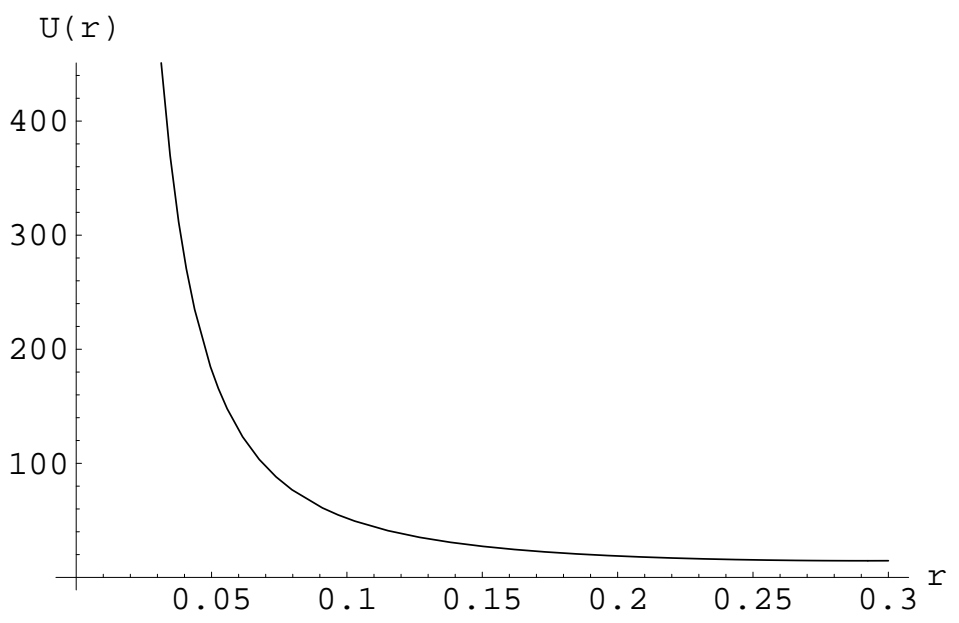

Figure 2: Potential corresponding to the model wave function with $\nu_{1}=2 / 9, \nu_{2}=-0.10$ and $R=-2$.

In addition to the above mechanism, the matrix elements of the local 6 quark operator in the $\boldsymbol{8}_{s}$ channel vanish in the non-relativistic limit, as shown in the previous section. Therefore the attraction in the $\boldsymbol{8}_{s}$ channel predicted by the OPE analysis can be hardly seen, as long as the non-relativistic approximation holds. Indeed this approximation seems good in the MC measurements 8 since the wave function in the $\mathbf{8}_{s}$ channel is observed to be very small at the origin.

\section{Conclusion}

In this paper we extended the OPE analysis of ref. [5] from two to three flavors, in order to investigate the short distance behavior of general baryon-baryon potentials in flavor $\mathrm{SU}(3)$. Interestingly the $\mathrm{OPE}$ analysis leads to manifest attraction for the $\mathrm{SU}(3)$ singlet potential at short distance, which is consistent with the quark model prediction and the lattice QCD result. The manifest attraction channel does not exist in the nucleon-nucleon potential for 2 flavors (the channels $\mathbf{2 7}_{s}$ and $\overline{\mathbf{1 0}}_{a}$ in the 3 flavor theory). The increase of the number of flavors decreases the repulsion at short distance and in some cases turns it into attraction already for 3 flavors, since the Pauli exclusion principle among quarks becomes less significant for larger number of flavors. The agreement among the three different methods strongly corroborates that the interaction between two baryons in the singlet channel is indeed attractive. It would be important to check by a lattice QCD calculation whether this attraction in the flavor SU(3) limit leads to a bound-state (the $\mathrm{H}$-particle). Even if the bound state is indeed present in the $\mathrm{SU}(3)$ limit, it is possible that it becomes a resonance above the $\Lambda \Lambda$ threshold in the real world where the strange quark is heavier than the other two.

On the other hand, our OPE analysis indicates that there is short distance attraction in both $\left(\mathbf{8}_{a}\right.$ and $\left.\boldsymbol{8}_{s}\right)$ octet channels, which disagrees with present lattice QCD results. Although we gave a possible explanation for this discrepancy, a direct confirmation by lattice QCD calculations will be needed for definite conclusions. 


\section{Acknowledgments}

S.A. would like to thank members of HAL QCD Collaboration, in particular, Prof. T. Hatsuda, Drs. Doi, Inoue, Ishii, Nemura and Sasaki, for useful discussions. S. A. is supported in part by Grant-in-Aid of the Ministry of Education, Sciences and Technology, Sports and Culture (Nos. 20340047, 20105001, 20105003). J. B. is grateful to the Center for Computational Sciences of the Tsukuba University for financial support and to the Particle Physics group for hospitality during his stay. This investigation was supported in part by the Hungarian National Science Fund OTKA (under K77400).

\section{References}

[1] N. Ishii, S. Aoki and T. Hatsuda, Phys. Rev. Lett. 99, 02201 (2007).

[2] S. Aoki, T. Hatsuda and N. Ishii, Comput. Sci. Dis. 1, 015009 (2008).

[3] S. Aoki, T. Hatsuda and N. Ishii, Prog. Theor. Phys. 123, 89 (2010).

[4] N. Ishii, S. Aoki and T. Hatsuda, PoS (Lattice 2008), 155 (2008)

[5] S. Aoki, J. Balog and P. Weisz, JHEP 1005, 008 (2010).

[6] M. Oka, K. Shimizu and K. Yazaki, Phys. Lett. B130, 365 (1983), Nucl. Phys. A464, 700 (1987), Prog. Theor. Phys. Suppl. 137, 1 (2000).

[7] C. Itzykson and M. Nauenberg, Rev. Mod. Phys. 38 (1966) 95.

[8] T. Inoue for HAL QCD Collaboration, PoS (Lattice 2009), 133 (2010).

T. Inoue et al., HAL QCD Collaboration, "Baryon-Baryon Interactions in the Flavor SU(3) Limit from Full QCD Simulations on the Lattice", [arXiv:1007.3559 [hep-lat]]. 
